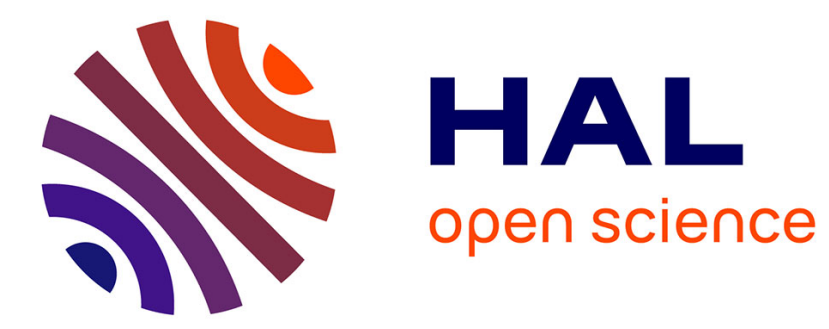

\title{
Premiers essais de l'appareil "Fossomatic" pour la détermination automatique du nombre de cellules du lait
}

R. Grappin, R. Jeunet

\section{To cite this version:}

R. Grappin, R. Jeunet. Premiers essais de l'appareil " Fossomatic "pour la détermination automatique du nombre de cellules du lait. Le Lait, 1974, 54 (539_540), pp.627-644. hal-00928671

\section{HAL Id: hal-00928671 \\ https://hal.science/hal-00928671}

Submitted on 1 Jan 1974

HAL is a multi-disciplinary open access archive for the deposit and dissemination of scientific research documents, whether they are published or not. The documents may come from teaching and research institutions in France or abroad, or from public or private research centers.
L'archive ouverte pluridisciplinaire HAL, est destinée au dépôt et à la diffusion de documents scientifiques de niveau recherche, publiés ou non, émanant des établissements d'enseignement et de recherche français ou étrangers, des laboratoires publics ou privés. 


\title{
Premiers essais de l'appareil "Fossomatic" pour la détermination automatique du nombre de cellules du lait
}

\author{
par \\ R. GRAPPIN et R. JEUNET \\ I.N.R.A., Station Expérimentale Laitière - 39800 Poligny
}

Actuellement, les laboratoires d'analyses disposent de deux appareils entièrement automatiques pour déterminer avec précision le nombre de cellules somatiques contenues dans le lait. Il s'agit de l'appareil Technicon OSCC II et de la version entièrement automatique du Compteur Coulter (Tolle et al., 1971 ; Grappin et Jeunet, 1973).

Ces deux appareils reposent sur des méthodes indirectes de numération des cellules du lait basées sur le comptage de particules en suspension dans un liquide. La précision de ces méthodes par rapport à la méthode microscopique a toujours été trouvée satisfaisante.

Selon un principe totalement différent, la Société danoise A/S N. Foss Electric a mis au point une méthode plus spécifique de comptage des cellules du lait, en utilisant une technique de dénombrement miscroscopique par fluorescence.

L'appareil, appelé Fossomatic, effectue de façon entièrement automatique, de la prise d'essai à l'inscription du résultat, la détermination du nombre des cellules du lait.

Nous donnons ici les premiers résultats obtenus avec cet appareil mis à notre disposition au mois de juillet 1974 par la Société A/S N. Foss Electric.

En raison des délais assez courts dont nous disposions pour faire cette étude, il ne nous a pas toujours été possible de procéder à tous les essais qu'il aurait été souhaitable de faire.

En fait, il ne s'agit pas ici d'une étude exhaustive de l'appareil Fossomatic, mais d'une série d'essais, parfois limités, destinés à obtenir un premier aperçu de la valeur de la méthode et de l'appareillage et à mettre en lumière certains problèmes qu'il conviendra d'étudier ultérieurement. 


\section{PRINCIPE}

Lorsque certains colorants sont soumis à une excitation lumineuse, leurs molécules ont la propriété d'émettre à leur tour une radiation lumineuse dont les longueurs d'onde sont plus élevées que les longueurs d'onde de la lumière absorbée : c'est le phénomène de fluorescence. Le colorant utilisé par l'appareil est du bromure d'éthidium ( $\left.\mathrm{C}_{21} \mathrm{H}_{3} \mathrm{Br} \mathrm{N}_{3} ; \mathrm{PM}=394,32\right)$. Lorsque ce colorant est mélangé au lait, il forme un complexe avec l'acide désoxyribonucléique (ADN) contenu dans le noyau des cellules. La bande de longueur d'onde utilisée pour le faisceau lumineux d'excitation est de 400-570 $\mathrm{nm}$ (lumière bleue), le maximum d'absorption du complexe ADN - bromure d'éthidium se situant à $520 \mathrm{~nm}$. L'émission de lumière fluorescente est limitée à l'aide d'un filtre interférentiel, à une bande de 530 à $700 \mathrm{~nm}$ (lumière rouge).

Une partie du colorant peut également se combiner avec les groupes phosphate de la membrane des globules gras mais le complexe formé émet de la lumière fluorescente à une longueur d'onde inférieure (vert-jaune) à celle émise par le complexe ADN-colorant. Par contre les bactéries qui absorbent également le colorant émettent une lumière rouge comme les cellules du lait. Toutefois, l'intensité lumineuse émise par ces bactéries ne peut atteindre un degré identique à celui des cellules du lait que s'il y a des amas microbiens. Aussi, l'appareil est-il pourvu d'un agitateur destiné à détruire ces amas.

\section{FONCTIONNEMENT DE L'APPAREIL}

\section{Dilution et coloration du lait}

Afin d'obtenir une fixation maximum du colorant par l'ADN, l'échantillon de lait prélevé $(0,2 \mathrm{ml})$ est dilué avec $1,8 \mathrm{ml}$ de solution tampon (1) de façon à obtenir un $\mathrm{pH}$ final proche de 5,8. Le lait dilué est ensuite mélangé avec $2 \mathrm{ml}$ de solution colorante (2).

La dilution finale du lait est de $1 / 20$.

Dilution et coloration se font automatiquement en quelques secondes à la température de $60^{\circ} \mathrm{C}-65^{\circ} \mathrm{C}$.

(1) Tampon : solution aqueuse comprenant 3,1 g/l d'hydrogénophtalate de potassium, $1,375 \mathrm{~g} / \mathrm{l}$ d'hydroxide de potassium et $1 \mathrm{ml}$ de solution aqueuse de Triton X 100 à 10 p. 100.

(2) Solution colorante : solution comprenant $20 \mathrm{ml}$ d'une solution aqueuse à 1 p. 1000 de bromure d'éthidium pour 11 de tampon. 


\section{Comptage des cellules}

Le comptage s'effectue de la manière suivante : le liquide est déposé, sous la forme d'un film de $0,5 \mathrm{~mm}$ de largeur et $10 \mu \mathrm{m}$ d'épaisseur, sur la surface périphérique d'un disque tournant à la vitesse de $60 \mathrm{t} / \mathrm{mn}$ devant l'objectif du microscope (fig. 1).

Après lecture, c'est-à-dire passage du film sous l'objectif, le disque est nettoyé avec un jet de solution de rinçage puis séché par de l'air chaud.

Le comptage est effectué en continu pendant $12 \mathrm{~s}$, (12 révolutions du disque), ce qui correspond à la lecture d'un volume de solution de $20 \mu \mathrm{l}$ (soit l'équivalent de $1 \mu \mathrm{l}$ de lait) étalé sur une bande de $3,50 \mathrm{~m}$.

La source lumineuse du microscope est une lampe à arc au xénon. Chaque cellule colorée passant sous l'objectif est soumise à la lumière bleue d'excitatioli et émet par fluorescence une certaine quantité d'énergie lumineuse de couleur rouge. Le faisceau fluorescent passe après grossissement au microscope au travers d'une fente de $150 \mu \mathrm{m}$ et arrive sur un tube photomultiplicateur qui le transforme en un signal électrique.

Ce signal est enregistré par un compteur électronique. En principe, si on admet qu'il y a une distribution au hasard des cellules dans le liquide et sur la surface de lecture, il ne doit passer qu'une seule cellule à la fois dans la fente de $150 \mu \mathrm{m}$; les problèmes de passage en coïncidence n'apparaissent que pour des laits ayant plus de 7 à 8 millions de cellules $/ \mathrm{ml}$.

Un dispositif de réglage du niveau de discrimination permet de ne compter que les impulsions supérieures à un certain seuil.

La durée totale d'une analyse est de $20 \mathrm{~s}$.

\section{ESSAIS DE L'APPAREIL}

\section{REPETABILITE ET PRECISION}

\section{Matériel et méthodes}

\section{Echantillons de lait examinés}

L'étude a porté sur 70 échantillons de lait choisis de façon à avoir une répartition à peu près uniforme des teneurs en cellules dans une gamme allant de 65000 à 2,5 millions de cellules par ml de lait. Les analyses ont été faites moins de $24 \mathrm{~h}$ après la traite sur du lait gardé à $4^{\circ} \mathrm{C}$ sans produit de conservation. 


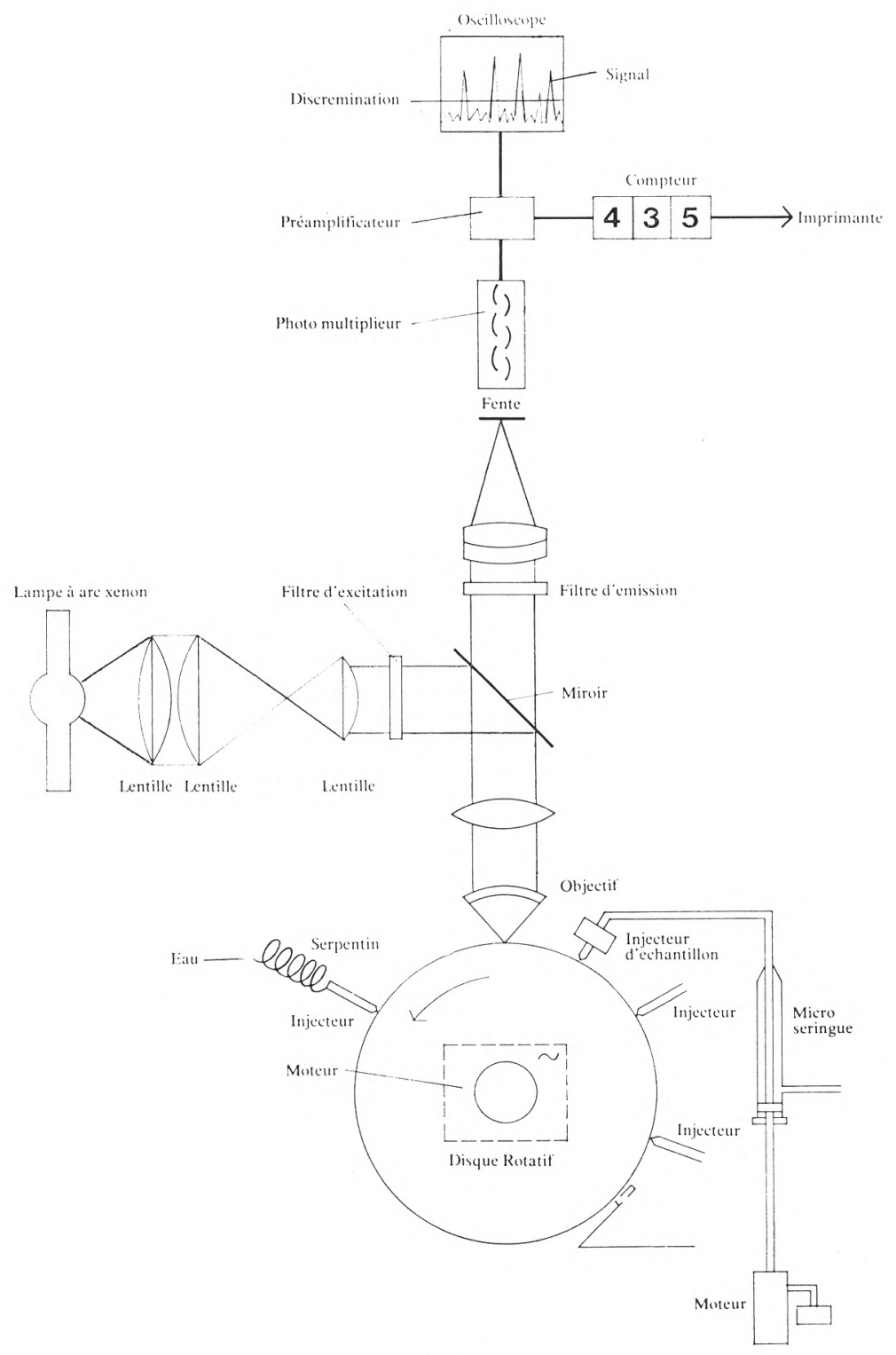

fig. 1

Schéma de fonctionnement des comptages microscopiques par l'appareil Fossomatic (doc. A/S N. Foss Electric) 


\section{Numération}

- Méthode microscopique : nous avons utilisé la méthode microscopique de numération de Prescott et Breed donnant le nombre de cellules par $\mathrm{ml}$ de lait (1).

Chaque échantillon de lait a fait l'objet de deux préparations microscopiques et, pour chaque préparation, on a effectué 40 lectures de champ. Le facteur microscopique employé est de 162000.

- Méthodes automatiques : chaque échantillon de lait a été analysé en double d'une part avec l'appareil Fossomatic et d'autre part, avec le Compteur Coulter. Le Fossomatic a été calibré au seuil de discrimination de 2,5 suivant les indications fournies par le constructeur (2).

Les numérations au Compteur Coulter ont été faites suivant la méthode préconisée par Tolle (Zeidler et al., 1968) en utilisant un appareil modèle F calibré à 4,6 $\mu \mathrm{m}$ (Grappin et Jeunet, 1971).

\section{Résultats}

\section{Répétabilité}

La répétabilité des mesures est estimée à partir du coefficient de variation des écarts arithmétiques entre résultats obtenus en double sur les mêmes échantillons. Nous l'avons calculée pour différents niveaux de concentration du lait en cellules (tab. 1).

Pour le Compteur Coulter et le Fossomatic, la répétabilité est meilleure lorsque les comptages sont élevés. La répétabilité du Fossomatic varie de 4,7 p. 100 pour les laits ayant moins de 250000 cellules $/ \mathrm{ml}$ à 2,5 p. 100 pour les laits ayant plus de 750000 cellules $/ \mathrm{ml}$.

$\mathrm{Au}$ niveau des comptages faibles, la répétabilité du Fossomatic apparaît, pour cet essai, meilleure que celle du Coulter. Dans une étude précédente (Grappin et Jeunet, 1973), les résultats obtenus avec le Compteur Coulter pour les laits contenant peu de cellules

(1) La composition du colorant utilisé est la suivante :

\begin{tabular}{|c|c|}
\hline - bleu de méthylène & $0,8 \mathrm{~g}$ \\
\hline - éosine & $0,2 \mathrm{~g}$ \\
\hline - alcool éthylique à $95^{\circ}$ & $54 \mathrm{ml}$ \\
\hline - tétrachloréthane & 40 \\
\hline - acide acétique glacial & 6 \\
\hline
\end{tabular}

(2) Signalons qu'en raison d'une mauvaise position de la canule de distribution du liquide sur le disque de lecture (injecteur d'échantillons) qui n'a été décelée qu'à la fin de l'essai, tous les résultats (x) donnés par l'appareil ont dû être modifiés selon une équation établie à partir de résultats obtenus sur des échantillons analysés avant et après que la position de cette canule ait été rectifiée (cette équation est la suivante : $\mathrm{y}=1,15 \mathrm{x}+25$ ). 
TABLEAU 1

Répétabilité des appareils Fossomatic et Compteur Coulter

\begin{tabular}{|c|c|c|c|c|c|c|}
\hline \multirow{2}{*}{$\begin{array}{l}\text { Niveau } \\
\text { (X 1000) }\end{array}$} & \multirow{2}{*}{$\mathrm{n}$} & \multirow{2}{*}{$\frac{\bar{x}}{x}$} & \multicolumn{2}{|c|}{ Fossomatic } & \multicolumn{2}{|c|}{ Coulter } \\
\hline & & & $\sigma$ & $\mathrm{C} \mathrm{V}$ & $\sigma$ & $\mathrm{C} \mathrm{V}$ \\
\hline$<\quad 250$ & 15 & 158 & 7,4 & 4,7 & 17,6 & 11,1 \\
\hline $250-500$ & 23 & 359 & 13,2 & 3,7 & 25,0 & 7,0 \\
\hline $500-750$ & 14 & 585 & 16,8 & 2,9 & 17,8 & 3,0 \\
\hline $750-2000$ & 13 & 1053 & 26,7 & 2,5 & 24,6 & 2,3 \\
\hline
\end{tabular}

$\mathrm{n}=$ nombre d'échantillons de lait.

$\overline{\mathrm{x}}=$ moyenne des comptages (en milliers de cellules $/ \mathrm{ml}$ ).

$\sigma=$ écart-type des écarts arithmétiques entre numérations faites en double (en milliers de cellules/ml).

$\mathrm{CV}=$ coefficient de variation (en p. 100).

étaient sensiblement meilleurs $(\mathrm{cv}=5,3 \mathrm{p}$. 100 pour une moyenne de 207000 cellules/ml).

\section{Précision}

Les résultats des comptages microscopiques d'une part (x) et avec le Fossomatic d'autre part (y) sont portés en échelle linéaire sur la figure 2. On constate que la précision de l'appareil, représentée par la dispersion des points autour de la droite de régression, augmente avec le nombre de cellules.

Le calcul de la variance résiduelle par niveau (tab. 2), indique que la précision suit une distribution de Poisson : la variance est approximativement proportionnelle à la moyenne des comptages. Pour estimer avec exactitude la corrélation entre les comptages microscopiques et les résultats du Fossomatic, on utilise une transformation racine carrée des données. Dans ces conditions, on obtient un coefficient de corrélation de 0,986 et un écart-type résiduel exprimé en racine carrée des valeurs de 1,33.

Le calcul approché de l'écart-type de la précision de l'appareil obtenu à partir de ce dernier résultat est voisin respectivement de 45,60 et 85000 cellules/ml aux différents niveaux de 250000 , 500000 et 1 million de cellules $/ \mathrm{ml}$. 


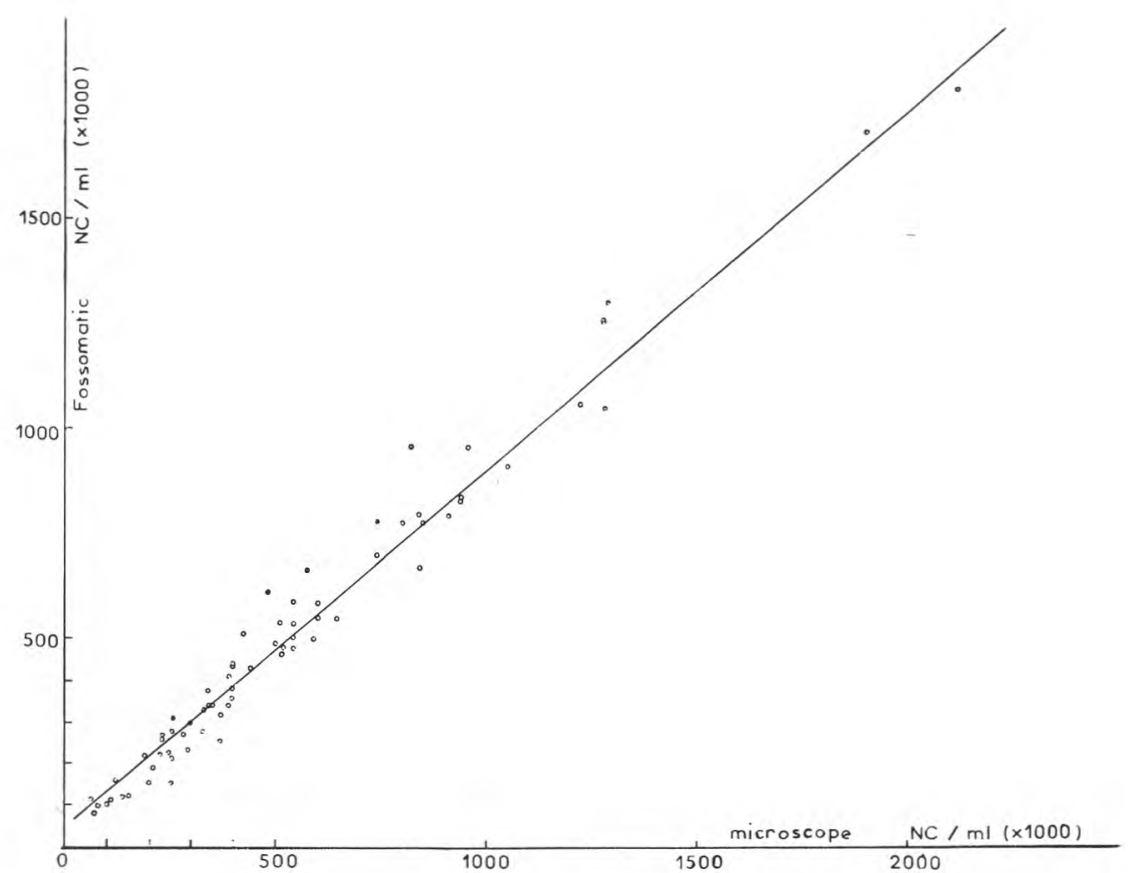

fig. 2

Relation entre les déterminations du nombre de cellules (NC) de 70 laits de troupeaux faites par la méthode microscopique $(x)$ et avec l'appareil Fossomatic (y).

$\mathrm{r}_{x, y}=0,987 ; \sigma_{y, x}=68000$ cellules $/ \mathrm{ml}$.

Dans le cas du Compteur Coulter, la précision des comptages au niveau des faibles teneurs en cellules $(<250000$ cellules) étant peu différente de celle qu'on observe aux autres niveaux (tab. 2), la transformation racine carrée ne s'impose pas.

Le coefficient de corrélation entre les comptages microscopiques et le Compteur Coulter calculé sur les données d'origine est de 0,976 (fig. 3). L'écart-type résiduel est égal à 73000 cellules $/ \mathrm{ml}$ quel que soit le niveau considéré (entre 100000 et 2 millions de cellules/ml). Ce résultat est moins bon que celui que nous avions obtenu précédemment (Grappin et Jeunet, 1973) avec $\mathrm{r}_{\mathrm{x}, \mathrm{y}}=0,990$ et $\sigma_{\mathrm{y}, \mathrm{x}}=58000$ cellules/ml.

La corrélation entre le Fossomatic et le Compteur Coulter calculée sur les nombres réels est de 0,985 (fig. 4) ; l'écart-type résiduel est de 64000 cellules/ml.

\section{Régression}

Compte tenu des modifications signalées plus haut, apportées aux résultats obtenus par le Fossomatic, on ne tiendra pas compte 
TABLEAU 2

Précision des appareils Fossomatic et Coulter par niveau

\begin{tabular}{|c|c|c|c|c|c|c|c|}
\hline \multirow{2}{*}{$\begin{array}{l}\text { Niveau } \\
\text { (X 1000) }\end{array}$} & \multicolumn{5}{|c|}{ Fossomatic } & \multicolumn{2}{|c|}{ Coulter } \\
\hline & $\mathrm{n}$ & $\bar{x}$ & $\sigma^{2}{ }_{s 1}, \mathrm{X}$ & $\sigma_{s 1}, \mathrm{X}$ & $\frac{\sigma^{2}{ }^{2}, \mathrm{X}}{\overline{\mathrm{x}}}$ & $\mathrm{n}$ & $\sigma_{\mathrm{y2}, \mathrm{X}}$ \\
\hline$<\quad 250$ & 15 & 158 & 904 & 30 & 5,7 & 15 & 65 \\
\hline $250-500$ & 23 & 361 & 2715 & 52 & 7,5 & 22 & 73 \\
\hline $500-1000$ & 23 & 700 & 4991 & 71 & 7,1 & 22 & 67 \\
\hline $1000-2500$ & 9 & 1566 & 10220 & 101 & 6,5 & 6 & 92 \\
\hline
\end{tabular}

Etude de la relation entre les comptages effectués d'une part par le Fossomatic $\left(\mathrm{y}_{1}\right)$ ou le Coulter $\left(\mathrm{y}_{2}\right)$ et d'autre part par la méthode microscopique $(\mathrm{x})$.

$\mathrm{n} \quad=$ nombre d'échantillons de lait.

$\overline{\mathrm{x}} \quad=$ moyenne arithmétique des comptages microscopiques (en milliers de cellules $/ \mathrm{ml}$ ).

$\sigma_{y i}, \mathrm{x}=$ écart-type résiduel déduit de la régression de $\mathrm{y}_{\mathrm{i}}$ sur $\mathrm{x}$ (en milliers de cellules/ml).

de la valeur du coefficient de régression $(b=0,846)$ obtenu dans cet essai. Cette question sera abordée dans le chapitre traitant du calibrage de l'appareil. La pente de la droite obtenue avec le Compteur Coulter est assez nettement inférieure à $1(\mathrm{~b}=0,750)$. Ce résultat confirme que le Coulter donne des résultats surestimés pour les échantillons contenant moins de 200 à 250000 cellules/ml.

\section{CALIBRAGE DE L'APPAREIL}

Dans le cas des analyses de lait, le calibrage ou étalonnage d'un appareil est une opération qui consiste à régler cet appareil de telle sorte qu'à tous les niveaux envisagés, la moyenne des mesures effectuées par l'appareil (y) sur différents laits soit égale ou très proche de la moyenne des mesures effectuées par une méthode de référence $(\mathrm{x})$. En d'autres termes, la droite de régression de y par rapport à $\mathrm{x}$ doit être de la forme $\mathrm{y}=\mathrm{bx}+\mathrm{a}$ avec $\mathrm{b}=1$ et $\mathrm{a}=\mathrm{O}$.

Dans le cas du Fossomatic, nous avons indiqué, en donnant le principe de l'appareil, que le calibrage de l'appareil se faisait en 


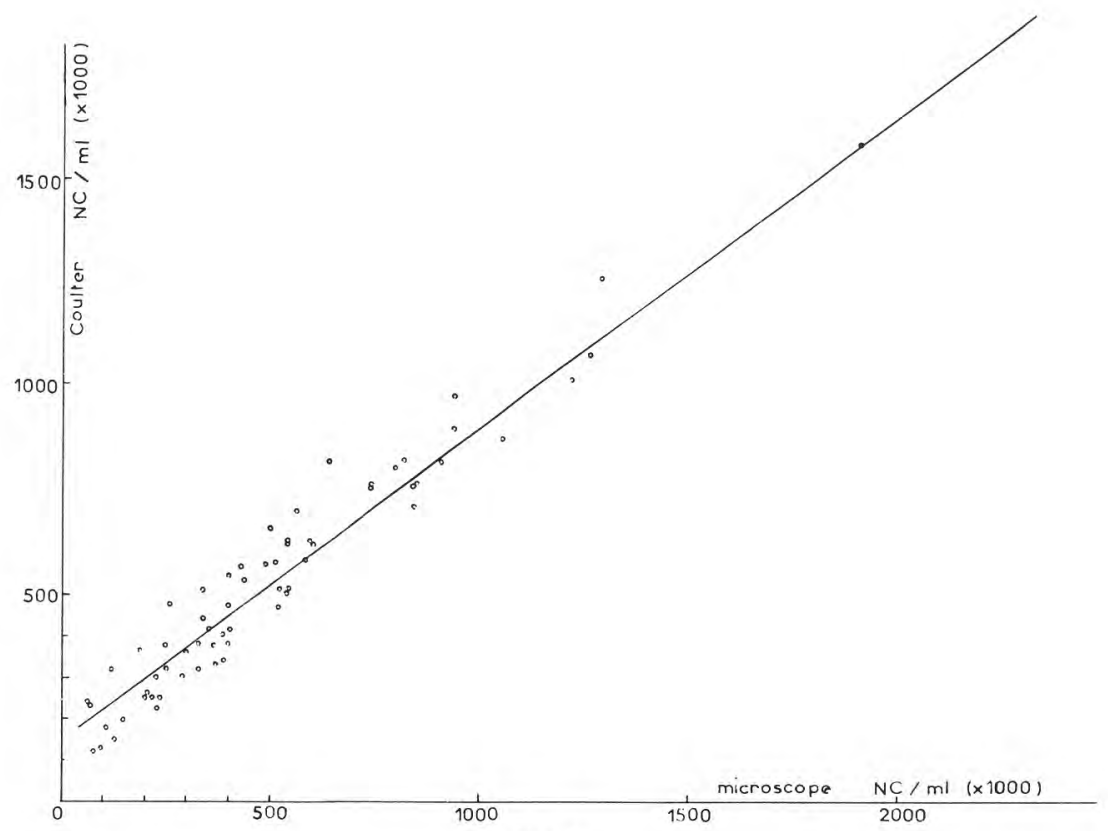

fig. 3

Relation entre les déterminations du nombre de cellules (NC) de 65 laits de troupeaux faites par la méthode microscopique (x) et avec l'appareil Coulter (y). $\mathrm{r}_{\mathrm{x}, \mathrm{y}}=0,976 ; \sigma_{y, \mathrm{x}}=73000$ cellules $/ \mathrm{ml}$.

réglant le seuil de discrimination. Le problème consiste donc à déterminer un seuil pour lequel on aura une équation de régression proche de l'équation théorique d'étalonnage.

Comment opérer ? Deux méthodes sont possibles : l'établissement d'une courbe de seuil et la comparaison avec des comptages microscopiques à différentes valeurs de seuil.

\section{Courbe de seuil}

Il y a théoriquement deux types d'impulsions susceptibles d'être comptées par l'appareil : des impulsions parasites ou « bruit » dont le niveau d'amplitude est relativement faible et des impulsions plus importantes provenant de la lumière fluorescente émise par les cellules du lait. Par conséquent, en analysant, aux différents seuils des échantillons de lait de teneur élevée en cellules, le diagramme cumulé des comptages devrait tout d'abord décroître au niveau du «bruit » puis présenter un plateau, et ensuite décroître au niveau des impulsions correspondant aux cellules du lait. Le seuil de lecture devrait se situer au milieu du plateau ; à ce niveau, l'appareil 


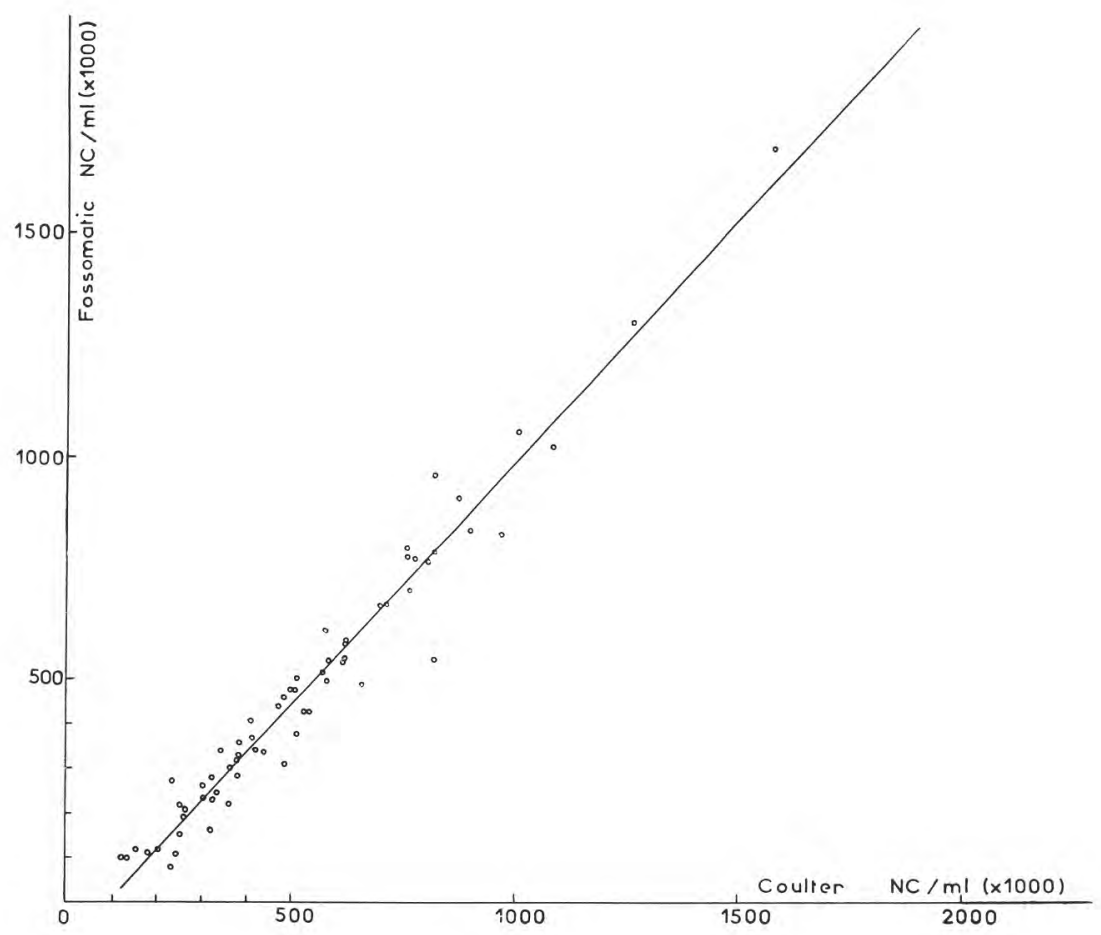

fig. 4

Relation entre les déterminations du nombre de cellules (NC) de 65 laits de troupeaux faites avec les appareils Compteur Coulter (x) et Fossomatic (y). $\mathrm{r}_{x, y}=0,985 ; \sigma_{y, x}=64000$ cellules $/ \mathrm{ml}$.

compte toutes les cellules sans tenir compte des impulsions parasites. En principe, en réglant l'appareil de cette manière, on devrait avoir des résultats très proches des résultats microscopiques.

En fait, toutes les courbes individuelles que nous avons réalisées montrent qu'il existe rarement un plateau bien marqué et que lorsque ce plateau existe, sa position varie d'un lait à un autre.

Afin d'obtenir une représentation moyenne, nous avons analysé simultanément 13 échantillons de lait frais renfermant entre 400000 et 6 millions de cellules/ml à différents seuils allant de 0,5 à 6,0 . Le nombre de cellules de ces échantillons a été déterminé par comptage microscopique. Sur la figure 5, sont représentés d'une part le diagramme de la moyenne géométrique des nombres cumulés en fonction du seuil ou " courbe de seuil " et d'autre part l'histogramme des nombres absolus par classe de seuil de 0,5. 


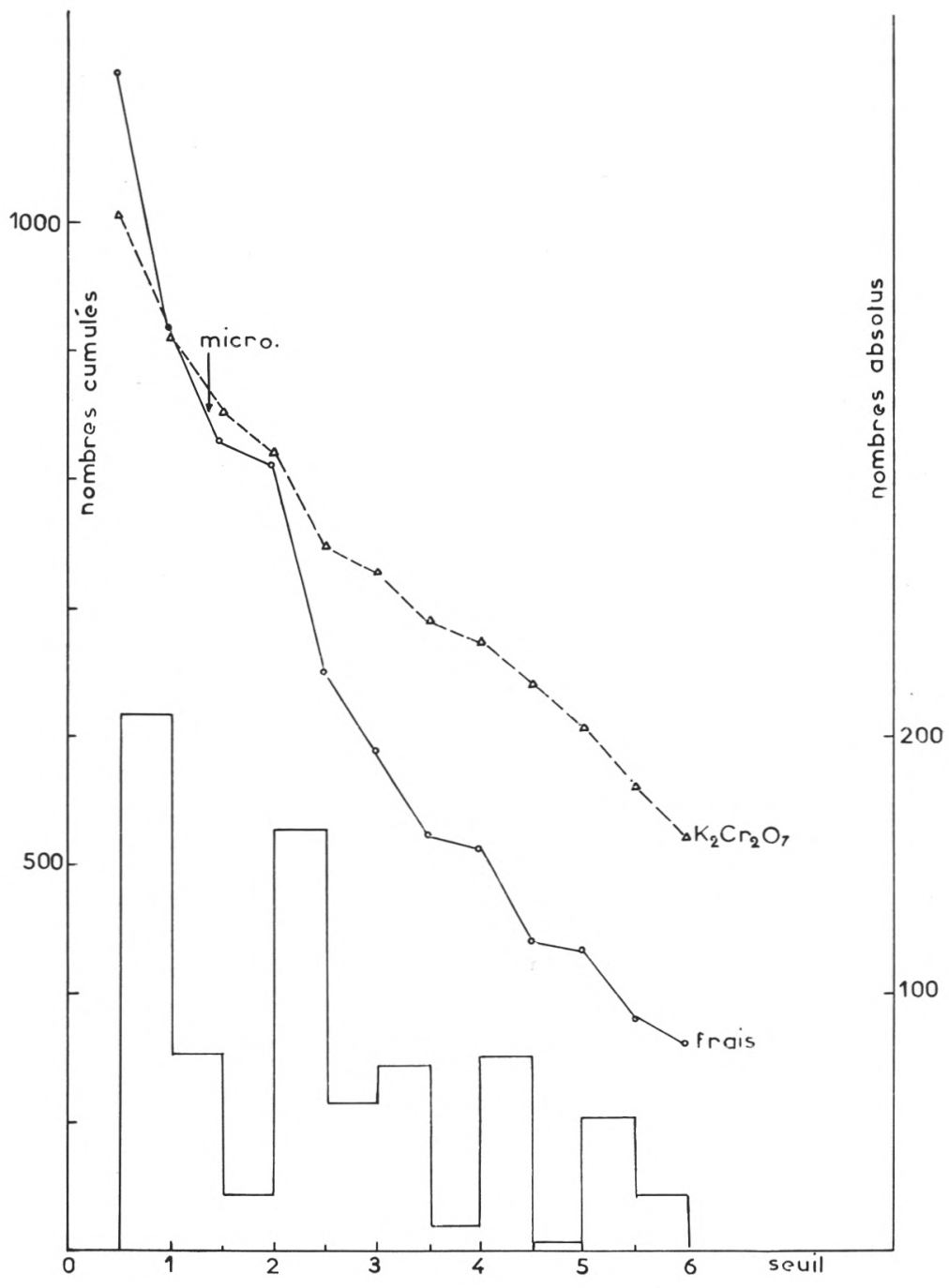

fig. 5

Distribution des comptages effectués par le Fossomatic en fonction des valeurs du seuil de discrimination :

- diagramme des moyennes géométriques des nombres cumulés des comptages effectués sur 13 échantillons de lait frais $(\mathrm{O}-\mathrm{O}-\mathrm{O})$ et additionnés de dichromate de potassium $(\triangle--\triangle--\triangle)$,

- histogramme des nombres absolus par classe de seuil. 
On constate que la courbe de seuil obtenue ne présente pas de plateau bien marqué. Il serait donc difficile de choisir, sur ce seul critère, le seuil de calibrage de l'appareil.

Si on se réfère à la moyenne géométrique des comptages microscopiques (moyenne égale à 857000 cellules $/ \mathrm{ml}$ ), on constate que le seuil de lecture devrait se situer vraisemblablement entre 1,0 et 1,5 (fig. 5). Mais en raison du petit nombre d'échantillons analysés, on ne peut obtenir ici qu'une indication approximative de la correspondance entre les comptages microscopiques et les comptages Fossomatic.

D'après ce résultat, le « bruit " serait inférieur au seuil de 1,0 et les impulsions provenant des cellules auraient une dimension supérieure au seuil de 1,5 ou 2,0. Si cette interprétation est exacte, cela signifie d'une part que le plateau est très réduit (pour cet essai, il se situerait entre 1,5 et 2,0 ) et d'autre part que l'intensité lumineuse émise par les cellules suit, d'après l'histogramme de la figure 5, une distribution à peu près lognormale.

Nous avons confirmé, dans un deuxième essai portant sur 18 échantillons de lait, la forme de cette distribution qui présente une dissymétrie gauche très nette.

\section{Comparaison avec des comptages microscopiques}

Cette deuxième méthode, plus exacte, consiste à effectuer, sur un assez grand nombre d'échantillons de lait (60 environ régulièrement répartis entre 100000 et 2 à 3 millions de cellules $/ \mathrm{ml}$ ) d'une part des comptages microscopiques et d'autre part des comptages avec le Fossomatic à différentes valeurs de seuil et à choisir le seuil pour lequel on obtient une pente de la droite de régression voisine de 1 et des moyennes égales, ainsi qu'un écart-type résiduel faible. C'est la technique qui a été employée pour définir le seuil de lecture du Compteur Coulter (Grappin et Jeunet, 1971) et qu'il conviendrait également d'utiliser avec le Fossomatic.

\section{INFLUENCE DES CONDITIONS DE CONSERVATION DES ECHANTILLONS DE LAIT}

\section{Nature du produit de conservation}

D'après le constructeur, il est possible d'analyser des échantillons conservés avec du dichromate de potassium. En revanche, le chlorure mercurique et le formol ne peuvent pas être employés.

A l'aide de plusieurs essais, nous avons testé l'influence des trois principaux produits de conservation utilisés pour les dosages chimiques du lait : le dichromate de potassium $\left(\mathrm{K}_{2} \mathrm{Cr}_{2} \mathrm{O}_{i}\right)$ à la dose de 1 p. 1000 ; le chlorure mercurique $\left(\mathrm{Hg} \mathrm{Cl} \mathrm{Cl}_{2}\right)$ à la dose de 0,7 p. 1000 et l'azide de sodium $\left(\mathrm{NaN}_{3}\right)$ à la dose de 0,3 p. 1000.

\section{Dichromate de potassium}

La série de 13 échantillons de lait utilisée pour définir la courbe de seuil (cf. plus haut) a également été analysée après addition de 
dichromate de potassium. Nous avons porté sur la figure 5 la courbe de seuil obtenue juste après addition de dichromate de potassium au lait. On constate une influence très nette du dichromate sur la forme de la courbe, par rapport à celle qui a été obtenue sur les mêmes échantillons de lait analysés sans conservateur. Si les deux courbes sont pratiquement confondues pour les valeurs de seuil comprises entre 1 et 2 , on note une pente nettement moins accentuée lorsque le lait est additionné de dichromate de potassium. Il s'en suit une augmentation très nette des comptages aux seuils supérieurs à 2 .

$\mathrm{Si}$ on admet que le seuil de lecture devrait être inférieur à 2, cela veut dire que l'addition du dichromate de potassium ne modifie pas les résultats donnés par l'appareil mais seulement la forme de la distribution du nombre de cellules.

Le dichromate agit-il sur la capacité de fixation du colorant par les cellules ou sur l'intensité de la lumière fluorescente émise par celles-ci ? C'est une question qui mériterait d'être approfondie.

\section{Azide de sodium}

L'analyse d'une série de 18 échantillons de lait a montré que la courbe de seuil obtenue après addition de l'azide n'est pas sensiblement différente de celle qui est obtenue avec les mêmes échantillons sans conservateur.

\section{Chlorure mercurique}

De nombreuses analyses effectuées au seuil de 3,5 sur des échantillons conservés avec du sublimé ont montré une augmentation très nette des comptages par rapport aux analyses faites sur les mêmes échantillons de lait sans conservateur. L'augmentation est du même ordre de grandeur que celle qui est constatée avec le dichromate de potassium.

\section{Durée et température de conservation}

Tous les essais concernant la conservation du lait ont été effectués au seuil de lecture de 3,5 qui paraissait, lorsque ces essais ont commencé, être le seuil de lecture optimum.

\section{Conservation de courte durée}

Deux séries d'échantillons de lait, l'une portant sur 10 échantillons et l'autre sur 13 ont été analysées d'une part le jour même de la traite et d'autre part après une nuit de conservation à $4^{\circ} \mathrm{C}$.

Chaque échantillon était réparti en deux flacons, un flacon sans produit de conservation et un autre avec du dichromate de potassium. Les résultats (tab. 3) montrent des augmentations surprenantes de 24 p. 100 (première série) et 37 p. 100 (deuxième série) des 
TABLEAU 3

Influence de la conservation des échantillons de lait à $4^{\circ} \mathrm{C}$ sur une courte période

\begin{tabular}{|c|c|c|c|c|}
\hline \multirow{2}{*}{$\begin{array}{c}\text { Analyses faites } \\
\text { au jour }\end{array}$} & \multicolumn{2}{|c|}{ Série $1(n=10)$} & \multicolumn{2}{|c|}{ Série $2(n=13)$} \\
\hline & $\begin{array}{c}\text { sans } \\
\text { conservateur }\end{array}$ & $\begin{array}{l}\mathrm{K}_{2} \mathrm{C}_{\mathrm{r} 2} \mathrm{O}_{7} \\
(1 \text { p. } 1000)\end{array}$ & $\begin{array}{c}\text { sans } \\
\text { conservateur }\end{array}$ & $\begin{array}{l}\mathrm{K}_{2} \mathrm{C}_{\mathrm{r} 2} \mathrm{O}_{7} \\
(1 \text { p. } 1000)\end{array}$ \\
\hline $\mathrm{J}$ & 356 & 418 & 521 & 695 \\
\hline $\mathrm{J}+1$ & $\begin{array}{c}442 \\
(+24 \text { p. } 100)\end{array}$ & $\begin{array}{c}498 \\
(+19 \text { p. } 100)\end{array}$ & $\begin{array}{c}714 \\
(+37 \text { p. } 100)\end{array}$ & $\begin{array}{c}757 \\
(+9 \text { p. 100) }\end{array}$ \\
\hline$J+2$ & $\begin{array}{c}466 \\
(+31 \text { p. } 100)\end{array}$ & $\begin{array}{c}506 \\
(+21 \text { p. } 100)\end{array}$ & & \\
\hline \multicolumn{5}{|c|}{$\begin{array}{l}\text { - moyenne géométrique des analyses faites par le Fossomatic, } \\
\text { - entre parenthèses : différence relative par rapport aux analyses faites le } \\
\text { jour du prélèvement (jour J). }\end{array}$} \\
\hline
\end{tabular}

résultats sur lait sans conservateur après une nuit de conservation. Les résultats obtenus sur les échantillons additionnés de dichromate ont augmenté de 19 p. 100 et 9 p. 100 .

\section{Conservation de longue durée}

Plusieurs séries de 18 échantillons additionnés de dichromate de potassium ont été analysées après différentes durées de conservation à $4^{\circ} \mathrm{C}$ ou $20^{\circ} \mathrm{C}$. Les résultats donnés sous la forme des moyennes géométriques sont résumés dans le tableau 4.

Il n'y a pas de modification très sensible des résultats après conservation des échantillons à $4^{\circ} \mathrm{C}$ pendant 2 semaines (séries 2 et 3) ou 3 semaines (série 1). En revanche, si les échantillons sont conservés à $20^{\circ} \mathrm{C}$, on constate une baisse très importante des résultats donnés par l'appareil au-delà de $3 \mathrm{j}$ de conservation (séries 2 , 3 et 4$)$.

\section{DISCUSSION}

Il est certain qu'en raison du principe utilisé, numération microscopique directe des cellules par fluorescence, le Fossomatic est un appareil séduisant. La répétabilité est satisfaisante et du même ordre de grandeur que celle des autres appareils automatiques de numération cellulaire (Technicon OSCC II et Compteur Coulter). 


\section{TABLEAU 4}

Influence de la durée de conservation des échantillons de lait additionnés de dichromate de potassium:

\begin{tabular}{|c|c|c|c|c|c|c|c|c|}
\hline \multirow{2}{*}{$\begin{array}{c}\text { Durée de } \\
\text { conservation }\end{array}$} & \multicolumn{2}{|c|}{ Série $1(1 / 7)$} & \multicolumn{2}{|c|}{ Série $2(5 / 7)$} & \multicolumn{2}{|c|}{ Série $3(8 / 7)$} & \multicolumn{2}{|c|}{ Série $4(12 / 7)$} \\
\hline & $4^{\circ} \mathrm{C}$ & $20^{\circ} \mathrm{C}$ & $4^{\circ} \mathrm{C}$ & $20^{\circ} \mathrm{C}$ & $4^{\circ} \mathrm{C}$ & $20^{\circ} \mathrm{C}$ & $4^{\circ} \mathrm{C}$ & $20^{\circ} \mathrm{C}$ \\
\hline 1 à $3 \mathrm{j}$ & 591 & & 395 & 395 & 360 & & 570 & 570 \\
\hline 4 à $7 \mathrm{j}$ & & & & & & & $\begin{array}{c}522 \\
(-8 \mathrm{p} .100)\end{array}$ & $\begin{array}{c}382 \\
(-33 \text { p. 100) }\end{array}$ \\
\hline 8 à $14 j$ & & & $\begin{array}{c}395 \\
(0 \text { p. } 100)\end{array}$ & $\begin{array}{c}118 \\
(-70 \text { p. } 100)\end{array}$ & $\begin{array}{c}349 \\
(-3 \text { p. 100) }\end{array}$ & $\begin{array}{c}207 \\
(-43 \text { p. } 100)\end{array}$ & & \\
\hline 15 à $21 \mathrm{j}$ & $\begin{array}{c}570 \\
(-4 \text { p. } 100)\end{array}$ & & & & & & & \\
\hline
\end{tabular}

- moyenne géométrique des analyses faites sur des séries de 18 échantillons,

- entre parenthèses : différence relative par rapport aux analyses faites entre 1 et $3 \mathrm{j}$ de conservation. 
La précision est également bonne, avec une mention toute particulière pour les comptages faibles $(<250000$ cellules $/ \mathrm{ml})$ qui se sont révélés être relativement très précis.

Le calibrage d'un appareil est un problème important. Bien que la méthode de la « courbe de seuil " soit justifiée sur le plan théorique, nos essais ont montré qu'il était difficile de l'utiliser pour réaliser un calibrage correct du Fossomatic. Il reste donc la comparaison avec la méthode microscopique.

Mais nos essais ne nous ayant pas permis de définir avec suffisamment de précision le niveau du seuil de discrimination auquel devront se faire les lectures, ce problème devra à nouveau être étudié.

On peut envisager de le résoudre de la façon suivante :

$1^{0}$ confier à plusieurs laboratoires spécialisés le soin de déterminer avec précision le seuil de lecture en se basant sur de nombreux comptages microscopiques,

$2^{\circ}$ ce seuil étant défini, il est indispensable de le relier soit à une grandeur caractéristique mesurable de l'appareil (par exemple, l'intensité de la lumière fluorescente au seuil défini), soit à un " échantillon étalon ", qui pourrait être composé de particules inertes fixant le colorant ou des suspensions de leucocytes, de concentration définie.

Avant d'envisager l'utilisation de cet appareil, il nous paraît indispensable que soit mis au point un système simple et précis de calibrage permettant de réaliser l'étalonnage initial des appareils et de contrôler leur bon fonctionnement, sachant qu'il est très difficile de demander aux utilisateurs de faire eux-mêmes des comptages microscopiques. Il faut en quelque sorte pouvoir assurer une bonne reproductibilité de la méthode.

L'étude de l'étalonnage ne doit pas être dissociée de celle de l'influence de la conservation des échantillons de lait. En effet, d'une part on a noté une augmentation des comptages après $24 \mathrm{~h}$ de conservation, et d'autre part nous avons montré que l'addition de dichromate de potassium modifiait très nettement les résultats donnés par l'appareil suivant le seuil auquel sont effectués les comptages, et ceci semble-t-il sans que la précision théorique des comptages en soit modifiée. Il peut également en être ainsi avec d'autres produits de conservation et avec des échantillons d'âge variable. Par conséquent, il n'est pas exclu que le seuil de lecture soit défini en fonction des conditions de conservation des échantillons de lait qui sont à analyser.

L'étude que nous avons faite a permis de mettre en évidence certains phénomènes liés à la conservation des échantillons de lait. Ce problème est particulièrement important dans le cas du Fossomatic car la plupart des utilisateurs seront à peu près certainement des laboratoires possédant déjà des combinés MTA/PMA et par 
conséquent, qui utiliseront les mêmes échantillons pour la numération leucocytaire et pour les dosages de matières azotées et de matière grasse. Or, dans la majorité des cas, les échantillons seront additionnés d'un produit de conservation et pourront lorsqu'il s'agira d'échantillons cumulés, être âgés de 15 j à 1 mois.

Sur le plan théorique, comme sur le plan pratique, l'appareil Fossomatic présente de nombreux atouts :

- il est basé sur une méthode spécifique de numération des cellules et donne des résultats précis et répétables, toyage,

- son emploi est simple, sans réglage journalier et sans net-

- la fiabilité mécanique, estimée sur une période de 5 semaines avec une utilisation moyenne de 2 à $3 \mathrm{~h}$ par jour, est bonne,

- les risques d'erreurs sont minimes, toutefois, comme pour tout appareil automatique, l'emploi régulier d'un " lait de contrôle » est indispensable,

- la préparation des réactifs est simple et la cadence d'analyses ne doit pas être très éloignée de la cadence théorique.

Mais actuellement, les modalités d'utilisation ne sont pas encore assez clairement définies. En particulier, le seuil de calibrage et la technique de calibrage doivent encore être étudiés. Certains aspects théoriques tels que la nature et l'importance des impulsions parasitaires ou " bruit " ne semblent pas encore assez bien connus. Quant aux conditions de conservation, de nouvelles études sont indispensables pour comprendre certains phénomènes mis en évidence au cours de ces essais et donner aux utilisateurs des éléments d'information précis.

\section{Rés u m é}

Des essais préliminaires ont été entrepris pour définir la valeur et les conditions d'utilisation de l'appareil Fossomatic de comptage automatique des cellules du lait.

La méthode de comptage microscopique des cellules par fluorescence, sur laquelle repose le principe de l'appareil permet d'obtenir des résultats satisfaisants : le coefficient de variation de répétabilité varie entre 4,7 et 2,5 p. 100 pour des laits dont la teneur en cellules varie de 150000 à 1 million de cellules $/ \mathrm{ml}$; l'écarttype de la précision par rapport aux comptages microscopiques est respectivement de 45000,60000 et 85000 cellules $/ \mathrm{ml}$ pour des niveaux de teneur en cellules de 250000 , 500000 et 1 million de cellules $/ \mathrm{ml}$ ( $\mathrm{n}=70$ échantillons).

Les résultats obtenus concernant le calibrage de l'appareil ainsi que les conditions de conservation des échantillons de lait (influence du produit de conservation, durée et température de conservation des échantillons) n'ont pas permis de donner des normes précises d'utilisation.

D’autres études de ces problèmes sont nécessaires. 


\section{S u m m ary}

Preliminary tests have been undertaken to assess the value and conditions of use of the Fossomatic instrument for automatic milk cells counting.

The fluorescent microscopic method of counting which is the basic principle of the instrument seems to give satisfactory results : the coefficient of variation of repeatability vary from 4.7 to 2.5 p. 100 for milk which cells content vary from 150,000 to 1 million of cells $/ \mathrm{ml}$; the standard deviation of accuracy, compared to the direct microscopic cell count method, is respectively of $45,000,60,000$ and 85,000 cells $/ \mathrm{ml}$ for levels of $250,000,500,000$ and 1 million of cells $/ \mathrm{ml}$ ( $\mathrm{n}=70$ samples).

The study of the calibration of the instrument and of the condition of preservation of the samples (influence of the kind of preservative, time and temperature of storage) have shown that these questions are relatively complex and need further experiments.

\section{Note}

Une nouvelle série d'essais complémentaires concernant l'étalonnage du Fossomatic et les conditions de conservation des échantillons venant d'être entreprise très récemment, il nous paraît utile de mentionner dès à présent, les résultats suivants :

$1^{\circ}$ Par comparaison avec la méthode microscopique, le seuil théorique de lecture doit se situer à une valeur de seuil comprise entre 1,2 et 1,5 .

$2^{\circ}$ De nombreuses courbes de seuil obtenues avec des échantillons de lait frais additionnés ou non de produit de conservation $\left(\mathrm{Hg} \mathrm{Cl}, \mathrm{Cr}_{2} \mathrm{O}_{3} \mathrm{~K}_{2}, \mathrm{NaN}_{3}\right)$ ont donné pour les différents types de conservation, des images semblables à celle de la figure 5 , c'est-à-dire que les courbes diffèrent les unes par rapport aux autres mais présentent des points d'intersection très proche du seuil théorique de lecture. Par conséquent, les laits additionnés de produits de conservation et notamment de $\mathrm{K}_{2} \mathrm{Cr}_{2} \mathrm{O}_{7}$ peuvent être analysés avec le Fossomatic.

3o Suivant l'état initial des échantillons et la nature du produit de conservation, les échantillons peuvent être analysés, sans modification significative des résultats, après 2-3 semaines de conservation à $4^{\circ} \mathrm{C}$ et $3-4 \mathrm{j}$ à $20^{\circ} \mathrm{C}$.

Les résultats détaillés de ces essais seront publiés ultérieurement.

19 novembre 1974.

\section{Références bibliographiques}

Tolle (A.), Heeschen (W.), Reichmuth (C.), Zeidler (H.) (1971), - Dairy Sci. Abstr., 33, 875-879.

Grappin (R.) et JeUnet (R.) (1971). - Le Lait, 51, 273-293.

GRAPPIn (R.) et Jeunet (R.) (1973). - Rev. Lait. Franc., 313, 737-747.

Zeidler (H.), Tolle (A.) und Heeschen (W.) (1968). - Milchwissenschaft, 23, 564-568. 